\title{
Synthèse et conceptualisation des processus cognitifs du jugement évaluatif de l'enseignant clinicien
}

\section{Synthesising and conceptualizing the cognitive processes of evaluative judgment by clinical teachers}

\author{
Geneviève GAUTHIER ${ }^{1}$, Christina ST-ONGE ${ }^{1}$, Valérie DORY ${ }^{2}$ \\ 1 Département de médecine, Centre de pédagogie des sciences de la santé, Faculté de médecine et des sciences de la santé. \\ Université de Sherbrooke (Canada) \\ 2 Centre d'éducation médicale, Université McGill, Montréal (Canada)
}

Manuscrit reçu le 22 avril 2017; commentaires éditoriaux formulés aux auteurs le 11 et le 26 mai 2017 ;

accepté pour publication le 26 mai 2017

Les articles commentés:

Gauthier G, St-Onge C, Tavares W. Rater cognition: review and integration of research findings. Med Educ 2016;50:511-522. ${ }^{1}$

St-Onge C, Chamberland M, Lévesque A, Varpio L. Expectations, observations, and the cognitive processes that bind them: expert assessment of examinee performance. Adv Health Sci Educ Theory Pract 2016;21:627642. ${ }^{2}$

Mots-clés
Évaluation ; jugement
évaluatif ;
raisonnement
évaluatif

Keywords
Rater cognition;
rater-based
assessment; cognitive
processes; judgment

Résumé - Contexte et problématique : Le recours accru au jugement évaluatif dans le contexte de l'évaluation des compétences a mené au développement du champ de recherche sur les processus cognitifs et le jugement des évaluateurs nommé "rater cognition ». Exégèse : Les auteurs analysent deux contributions qui proposent respectivement une modélisation du raisonnement évaluatif, issue soit des écrits scientifiques soit de données empiriques. Ces modélisations illustrent, de façon complémentaire, la complexité du jugement évaluatif et suggèrent différentes avenues pour l'optimisation de certaines pratiques évaluatives.

\begin{abstract}
Background: The increased reliance on competency-based framework and raterbased judgment has led to the development of research on cognitive processes and the judgment of evaluators called "rater cognition". Findings: Authors analyze two contributions on conceptual models of evaluative judgment resulting either from scientific writings or empirical data. The modeling illustrates in a complementary way the complexity of evaluative judgments and suggests different avenues to optimize a number of evaluative practices.
\end{abstract}




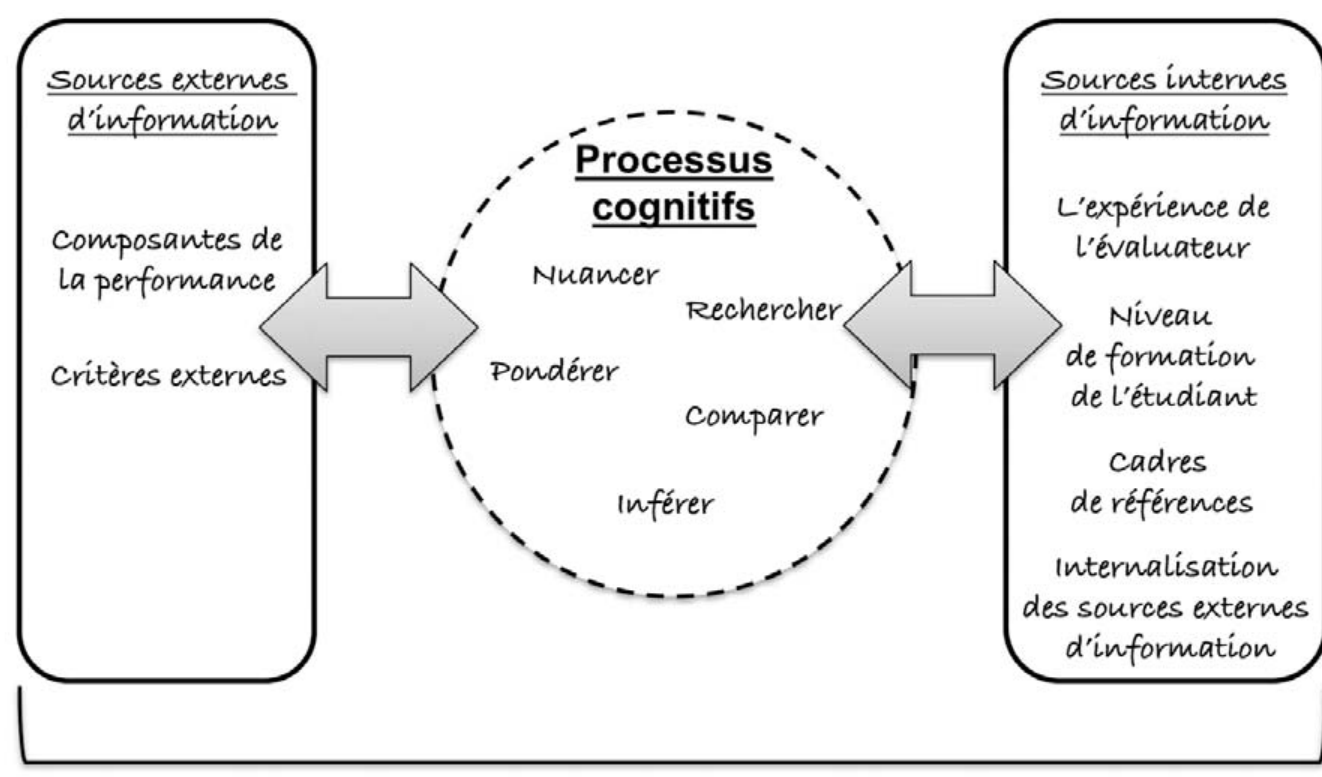

Évaluation significative

Fig. 1. Modèle conceptuel des processus cognitifs en jeu pour une évaluation significative selon St-Onge et al. ${ }^{\text {[2] }}$

\section{Problématique}

Le recours à des approches pédagogiques centrées sur l'acquisition de compétences est de plus en plus présent en pédagogie des sciences de la santé ${ }^{[3 \text {, }}$ ${ }^{4]}$. Ces approches pédagogiques ont entraîné des changements en ce qui concerne les pratiques évaluatives, notamment en privilégiant l'évaluation de la performance des apprenants lors de l'exécution de tâches complexes ${ }^{[5]}$. Ces pratiques nécessitent le recours à des évaluateurs dont la variabilité du jugement est bien documentée ${ }^{[6]}$. Ce recours accru au jugement évaluatif dans le contexte de l'évaluation des compétences a mené au développement du champ de recherche sur le jugement des évaluateurs, tout particulièrement sur les processus cognitifs en jeu (« rater cognition »).

Jusqu'à maintenant, les recherches effectuées sur l'évaluation de la performance en contexte de pédagogie des sciences de la santé se sont focalisées sur les évaluations à enjeux élevés (par exemple les examens de certification). Traditionnellement, ces études ont été réalisées selon une perspective psychométrique, visant à limiter l'erreur de mesure liée à la subjectivité des évaluateurs ${ }^{[7,8]}$. Plus récemment, certains chercheurs ont commencé à explorer les différents facteurs à l'origine de la variabilité des évaluateurs ${ }^{[9,10]}$ ainsi que la nature de l'expertise des évaluateurs cliniciens expérimentés ${ }^{[11-13]}$. Nous faisons état ici de deux articles, utilisant l'un une approche empirique et l'autre une approche théorique, qui ont tenté d'articuler la complexité des processus cognitifs liée à l'évaluation de la performance clinique.

\section{Ce que les auteurs ont fait}

Le tableau I résume les grandes lignes méthodologiques des articles de St-Onge et al. ${ }^{[2]}$ et de Gauthier et al. ${ }^{[1]}$ en termes de : 1) dispositif méthodologique ; 2) identification des articles / recrutement des participants ; 3) collecte des données et 4) analyse des données pour mieux contextualiser leurs résultats de recherche. 
Tableau I. Résumé des méthodologies employées dans les deux articles commentés.

\begin{tabular}{|c|c|c|}
\hline & St-Onge et al. ${ }^{[2]}$ & Gauthier et al. $^{[1]}$ \\
\hline $\begin{array}{l}\text { Dispositif } \\
\text { méthodologique }\end{array}$ & $\begin{array}{l}\text { Théorisation ancrée à partir d'entrevues } \\
\text { semi-dirigées réalisées avec des cliniciens } \\
\text { lors de l'évaluation d'une performance } \\
\text { complexe d'un résident avec un patient } \\
\text { standardisé }\end{array}$ & Article de synthèse \\
\hline $\begin{array}{l}\text { Stratégie } \\
\text { d'identification des } \\
\text { articles / de } \\
\text { recrutement des } \\
\text { participants }\end{array}$ & $\begin{array}{l}\text { Identification d'évaluateurs experts par les } \\
\text { directeurs de programmes, membres du } \\
\text { corps professoral, ainsi que les résidents } \\
\text { (méthodologie décrite en détail dans St- } \\
\text { Onge et al. }{ }^{[14]} \text { ) }\end{array}$ & 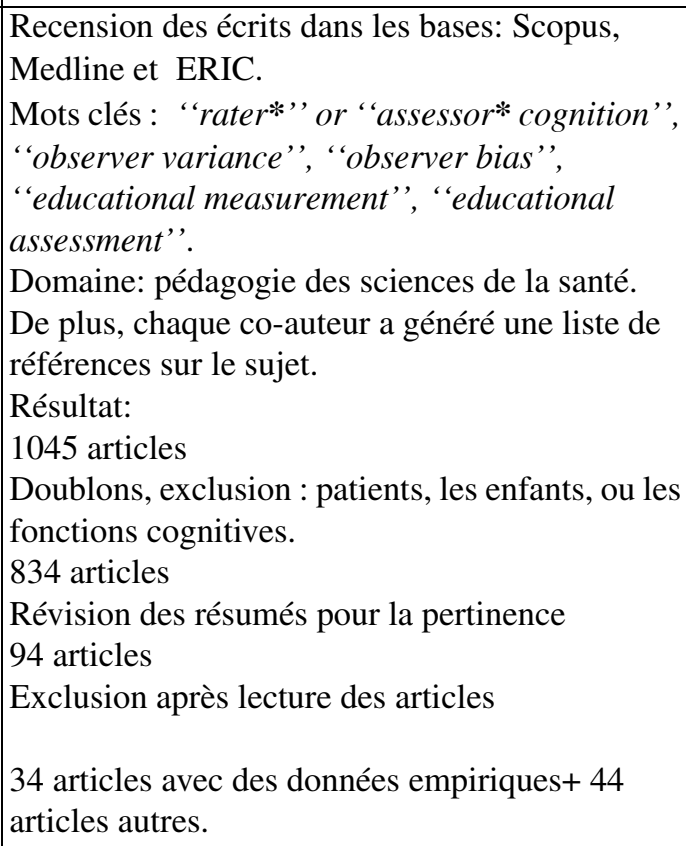 \\
\hline Collecte de données & $\begin{array}{l}\text { Entrevues semi-structurées avec } \\
11 \text { cliniciens enseignants qui observaient } \\
\text { un vidéo d'une résidente avec patient } \\
\text { standardisé et qui devaient verbaliser leur } \\
\text { évaluation de cette performance complexe. }\end{array}$ & $\begin{array}{l}\text { Extraction des données par la chercheure } \\
\text { principale avec retours fréquents auprès d'une } \\
\text { des co-auteurs (CSTO) pour validation du } \\
\text { processus. }\end{array}$ \\
\hline Analyse & $\begin{array}{l}\text { Identification et définition de thèmes } \\
\text { émergents quant aux processus cognitifs } \\
\text { des évaluateurs en contexte d'évaluation } \\
\text { d'une performance clinique, et ce, dans le } \\
\text { cadre d'un processus itératif de codage et } \\
\text { d'analyse. }\end{array}$ & $\begin{array}{l}\text { Identification et définitions de thèmes émergents } \\
\text { quant à la terminologie utilisée ainsi que la } \\
\text { description de mécanismes ou conceptualisations } \\
\text { de ces termes à travers les différents articles. }\end{array}$ \\
\hline
\end{tabular}

\section{Ce que les auteurs ont trouvé}

Les résultats de l'étude de St-Onge et al. ${ }^{[2]}$ illustrent très bien la multi dimensionnalité et la complexité du processus d'évaluation d'une performance dite complexe. Plus spécifiquement, cette équipe a observé que les évaluateurs doivent jongler avec plusieurs éléments, notamment des sources internes (par exemple : leur conceptualisation de ce qu'est la compétence attendue pour un étudiant d'un niveau donné) et externes d'informations (par exemple : les jalons ou balises des organismes certificateurs tel que le Collège royal des médecins et chirurgiens du Canada). Qui plus est, les évaluateurs ont recours, de façon plus ou moins structurée ou explicite, à une multitude de processus cognitifs leur permettant de 
Tableau II. Mécanismes en jeu lors de la phase d'observation des performances dans le cadre d'une démarche évaluative, selon Gauthier et al. ${ }^{[1]}$

\begin{tabular}{|l|l|}
\hline Phase 1: Observation & Brève description des mécanismes \\
\hline $\begin{array}{l}\text { Génération automatique } \\
\text { d'impressions des personnes }\end{array}$ & $\begin{array}{l}\text { Biais cognitif de généralisation (effet de halo) où des aspects sociaux ou des } \\
\text { traits personnels influencent inconsciemment le jugement }\end{array}$ \\
\hline $\begin{array}{l}\text { Formulation d'inférences de haut } \\
\text { niveau }\end{array}$ & $\begin{array}{l}\text { Inférences à propos des caractéristiques ou des compétences qui sont basées sur } \\
\text { d'autres faits qui ne sont pas articulés, justifiés ou observables et qui diffèrent } \\
\text { selon les évaluateurs }\end{array}$ \\
\hline $\begin{array}{l}\text { Mettre l'accent sur différentes } \\
\text { dimensions des compétences }\end{array}$ & $\begin{array}{l}\text { Attention portée à différents éléments d'une performance en fonction d'une } \\
\text { compréhension différente des composantes de compétences }\end{array}$ \\
\hline
\end{tabular}

Tableau III. Mécanismes en jeu lors de la phase de traitement de l'information dans le cadre d'une démarche évaluative, selon Gauthier et al. ${ }^{[1]}$

\begin{tabular}{|l|l|}
\hline Phase 2: Traitement de l'information & Brève description des mécanismes \\
\hline Catégorisation de l'information à travers des schémas basés sur: \\
\hline Le concept personnel de compétence & $\begin{array}{l}\text { Englobe : 1) une composante expérientielle ; 2) des éléments de conception } \\
\text { personnelle de bonne performance ; 3) des références externes intériorisées } \\
\text { et : 4) des définitions opérationnelles variées des compétences ciblées }\end{array}$ \\
\hline $\begin{array}{l}\text { La comparaison avec des schémas } \\
\text { d'exemples de provenance variée }\end{array}$ & $\begin{array}{l}\text { Utilisation d'exemples en lien avec les étudiants précédents, soi-même, des } \\
\text { collègues, d'anciens étudiants, pour établir des points de référence et des } \\
\text { standards pour comparer des composantes de la performance }\end{array}$ \\
\hline La spécificité de la tâche et du contexte & $\begin{array}{l}\text { La compréhension de la nature d'une tâche spécifique et de ses contraintes } \\
\text { sur la performance se développe à travers l'expérience d'évaluation, à } \\
\text { laquelle s'ajoutent les buts et le contexte de l'évaluation qui, eux, jouent un } \\
\text { rôle d'agents médiateurs sur l'évaluation }\end{array}$ \\
\hline
\end{tabular}

traiter les informations à leur disposition. Ce processus sollicite des tensions constantes quant à la perception de la subjectivité ou de l'objectivité de l'évaluation, et requiert parallèlement des habiletés spécifiques pour pondérer et nuancer les informations disponibles. Ces processus permettent aux évaluateurs de tenir compte à la fois de données externes et de données internes pour générer un jugement nuancé sur la performance observée.

D'autres études empiriques, issues de courants de recherche divers, ont été synthétisées par Gauthier et al. ${ }^{[1]}$, leur permettant de dégager un cadre conceptuel du jugement évaluatif. Ils ont regroupé en trois phases et neuf mécanismes les éléments communs aux différents écrits recensés sur le jugement évaluatif. La première phase, celle d'observation qui est décrite au tableau II, fait référence aux processus actifs (et non passifs) de perception et de sélection d'informations concernant la performance. Dans cette phase les auteurs présentent trois mécanismes qui influencent l'ensemble du processus cognitif d'observation.

La deuxième phase, celle du traitement de l'information qui est décrite au tableau III, fait référence à un processus de catégorisation implicite et automatique à travers lequel les évaluateurs accèdent et adaptent des schémas complexes d'exemples de performances antérieures qu'ils combinent implicitement à leurs propres conceptualisations de performance pour établir des critères adaptés à la spécificité de la tâche et du contexte.

La troisième phase, celle de l'intégration de l'information qui est décrite au tableau IV, inclut la combinaison des différentes sources d'informations 
Tableau IV. Mécanismes en jeu lors de la phase d'intégration de l'information dans le cadre d'une démarche évaluative, selon Gauthier et al. ${ }^{[1]}$

\begin{tabular}{|l|l|}
\hline Phase 3 : Intégration & Brève description des mécanismes \\
\hline $\begin{array}{l}\text { Stratégies de pondération et de } \\
\text { synthétisation de l'information }\end{array}$ & $\begin{array}{l}\text { Ces stratégies sont diverses et variées ; elles incluent une pondération égale de } \\
\text { toutes les sources d'information dont les évaluateurs sont conscients, une } \\
\text { priorisation par rapport à l'importance relative des composantes d'une } \\
\text { compétence donnée ou encore des aspects d'un comportement qui équivalent à } \\
\text { un échec, ou d'une perte de confiance face au raisonnement de l'étudiant }\end{array}$ \\
\hline $\begin{array}{l}\text { Production de jugement en forme } \\
\text { narrative }\end{array}$ & $\begin{array}{l}\text { Le jugement se développe en forme narrative et les demandes de comparaison } \\
\text { et de catégorisation des performances complexes créent beaucoup } \\
\text { d'incertitude chez les évaluateurs }\end{array}$ \\
\hline $\begin{array}{l}\text { Traduction du jugement narratif en } \\
\text { chiffre pour une grille d'évaluation }\end{array}$ & $\begin{array}{l}\text { La traduction d'un jugement global en description narrative qui est } \\
\text { subséquemment traduite en pointage pour les différentes échelles d'une grille } \\
\text { d'évaluation }\end{array}$ \\
\hline
\end{tabular}

pour produire un jugement en s'appuyant sur l'observation et l'interprétation de la performance d'un étudiant.

\section{Ce que les auteurs proposent comme retombées pédagogiques}

Si ces articles n'offrent pas de solutions précises pour pallier la variation de jugements pour l'évaluation de performances complexes, ils permettent de mettre en lumière la complexité cognitive et la nature contextuelle du jugement évaluatif. Cette recension et cette documentation d'un nombre non exhaustif de mécanismes cognitifs interagissant lors du processus de jugement d'une performance offrent des mots permettant de nommer les différentes composantes du jugement, par exemple lorsqu'il s'agit d'avoir une conversation à propos du jugement des évaluateurs à l'aide d'un cadre conceptuel. Ce cadre peut être utilisé pour mieux comprendre et étudier le phénomène dans un contexte où l'évaluateur et son expertise - et non la fiche d'évaluation - sont considérés comme l'outil d'évaluation.

Plus spécifiquement, Gauthier et al. ${ }^{[1]}$ et St-Onge et al. ${ }^{[2]}$ mettent en relief la nature complexe et multidimensionnelle du processus évaluatif lors d'une évaluation de performance complexe. Ces écrits pourraient être utilisés comme base pour élaborer une formation professorale qui aurait comme objectif de rendre plus explicite le processus évaluatif qui demeure très implicite pour les professeurs cliniciens. Lajoie ${ }^{[15]}$ stipule que rendre le processus ou la trajectoire explicites permet aux novices d'acquérir leur expertise plus rapidement. Ainsi, rendre explicite le processus évaluatif d'évaluateurs chevronnés pourrait permettre aux novices de structurer leurs propres processus et d'acquérir une certaine expertise plus rapidement.

Gauthier et al. ${ }^{[1]}$, ainsi que Kogan ${ }^{[13]}$ et Yeates et al. ${ }^{[16]}$ soulignent l'effet délétère que peut avoir la « traduction d'une évaluation » en « chiffre ». Cette étape du processus évaluatif peut faire perdre du sens et dénaturer l'évaluation de la performance des étudiants. D'autres études sont requises pour qualifier et quantifier les conséquences potentiellement négatives de cette pratique forçant les évaluateurs à chiffrer une performance ; toutefois les instances responsables de l'agrément au Canada (Committee On Accreditation Of Canadian Medical Schools - CACMS-; Royal College of Physicians and Surgeons of Canada-RCPSC) ont déjà instauré de nouvelles normes qui requièrent que les apprenants reçoivent des évaluations dites narratives et non seulement des évaluations chiffrées. 


\section{Perspectives de recherche}

La plupart des études recensées par Gauthier et al. ${ }^{[1]}$ se centrent, comme celle de St-Onge et al. ${ }^{[2]}$, sur une occasion particulière d'évaluation. Or en supervision clinique, le clinicien enseignant est généralement en contact prolongé avec l'apprenant et peut dès lors non seulement combiner diverses observations, mais cheminer dans un raisonnement qui se raffine dans le temps, où une première observation peut donner lieu à plusieurs hypothèses pédagogiques, qu'il vérifiera ensuite par un recueil de données proactif et ciblé ${ }^{[17]}$. Ce champ de recherche en est encore à ses balbutiements. De nombreux chantiers sont ouverts ou restent à entamer. Les recherches sur le jugement évaluatif illustrent le parallèle entre celuici et le raisonnement clinique. Comme la majorité des recherches sur le raisonnement clinique, elles se fondent sur des théories cognitivistes ${ }^{[18]}$. Ces théories font généralement peu de place à des aspects liés par exemple aux émotions ${ }^{[19,20]}$ ou au contexte social ${ }^{[18 \text {, }}$ ${ }^{21]}$. A terme, la prise en compte et l'exploration empirique de ces facteurs devraient enrichir le cadre conceptuel proposé.

\section{Contributions}

Geneviève Gauthier et Christina St-Onge on écrit l'ébauche initiale pour le résumé des articles et Valérie Dory a critiqué et écrit les limites et perspectives de recherche. Toutes les auteures ont contribué au raffinement, à la réécriture et à la révision de l'article. Toutes les auteures ont approuvé la version finale.

\section{Liens d'intérêts}

Aucune auteure ne déclare de conflit d'intérêt en lien avec le contenu de cet article.

\section{Approbation éthique}

Sans objet

\section{Références}

1 Gauthier G, St-Onge C, Tavares W. Rater cognition: review and integration of research findings. Med Educ 2016;50:511-522.

2 St-Onge C, Chamberland M, Lévesque A, Varpio L. Expectations, observations, and the cognitive processes that bind them: expert assessment of examinee performance. Adv Health Sci Educ Theory Pract 2016;21:627-642.

3 Jouquan J. L'évaluation des apprentissages des étudiants en formation médicale initiale. Pédagogie Médicale 2002;3:38-52.

4 Howley LD. Performance assessment in Medical Education: where we've been and where we're going. Eval Health Prof 2004;27:285-303.

5 Whitehead CR, Kuper A, Hodges B, Ellaway R. Conceptual and practical challenges in the assessment of physician competencies. Med Teach 2015;37:245251.

6 Downing SM. Threats to the validity of clinical teaching assessments: what about rater error? Med Educ 2005;39:353-355.

7 Norcini J, Burch V. Workplace-based assessment as an educational tool: AMEE Guide No. 31. Med Teach 2007;29:855-871.

8 Williams RG, Klamen DA, McGaghie WC. Cognitive, social and environmental sources of bias in clinical performance ratings. Teach Learn Med 2003;15:270292.

9 Gingerich A, Kogan J, Yeates P, Govaerts M, Holmboe E. Seeing the 'black box' differently: assessor cognition from three research perspectives. Med Educ 2014;48:1055-1068.

10 Essers G, van Dulmen S, van Weel C, van der Vleuten $\mathrm{C}$, Kramer A. Identifying context factors explaining physician's low performance in communication assessment: an explorative study in general practice. BMC Fam Pract 2011;12:1-8.

11 Govaerts MJB, Schuwirth LWT, van der Vleuten CPM, Muijtjens AMM. Workplace-based assessment: effects of rater expertise. Adv Health Sci Educ Theory Pract 2011;16:151-165.

12 Berendonk C, Stalmeijer R, Schuwirth LT. Expertise in performance assessment: assessors' perspectives. Adv Health Sci Educ Theory Pract 2013;18:559-571. 
13 Kogan JR, Conforti L, Bernabeo E, Iobst W, Holmboe E. Opening the black box of clinical skills assessment via observation: a conceptual model. Med Educ 2011;45:1048-1060.

14 St-Onge C, Chamberland M, Lévesque A, Varpio L. The role of the assessor: exploring the clinical supervisor's skill set. The Clinical Teacher 2014;11:209213.

15 Lajoie SP. Transitions and trajectories for studies of expertise. Educ Res 2003;32:21-25.

16 Yeates P, O’Neill P, Mann K, Eva K. Seeing the same thing differently: mechanisms that contribute to assessor differences in directly-observed performance assessments. Adv Health Sci Educ Theory Pract 2013; 18:325-341.

17 Audétat M-C, Laurin S, Sanche G. Aborder le raisonnement clinique du point de vue pédagogique - I. Un cadre conceptuel pour identifier les problèmes de raisonnement clinique. Pédagogie Médicale 2011;12: 223-229.
18 Durning SJ, Artino ARJ, Schuwirth L, van der Vleuten C. Clarifying assumptions to enhance our understanding and assessment of clinical reasoning. Acad Med 2013;88:442-448.

19 Gomez-Garibello C, Young M. Emotions and assessment: examining rater-based judgements of entrustment. Soumis.

20 Weber EU, Johnson EJ. Mindful judgment and decision making. Annu Rev Psychol 2009;60:53-85.

21 Kogan JR, Conforti LN, Bernabeo E, Iobst W, Holmboe E. How faculty members experience workplacebased assessment rater training: a qualitative study. Med Educ 2015;49:692-708.

Correspondance et offprints : Geneviève Gauthier. Département de médecine, Centre de pédagogie des sciences de la santé, Faculté de médecine et des sciences de la santé. Université de Sherbrooke, 3001-12e Avenue Nord, J1H 5N4 Sherbrooke, Québec, Canada 